\title{
3D Space-charge model for GPT simulations of high- brightness electron bunches
}

Citation for published version (APA):

Geer, van der, S. B., Luiten, O. J., Loos, de, M. J., Pöplau, G., \& Rienen, van, U. (2003). 3D Space-charge model for GPT simulations of high-brightness electron bunches. (TESLA report; Vol. 2003-04). DESY.

Document status and date:

Published: 01/01/2003

\section{Document Version:}

Publisher's PDF, also known as Version of Record (includes final page, issue and volume numbers)

\section{Please check the document version of this publication:}

- A submitted manuscript is the version of the article upon submission and before peer-review. There can be important differences between the submitted version and the official published version of record. People interested in the research are advised to contact the author for the final version of the publication, or visit the $\mathrm{DOI}$ to the publisher's website.

- The final author version and the galley proof are versions of the publication after peer review.

- The final published version features the final layout of the paper including the volume, issue and page numbers.

Link to publication

\section{General rights}

Copyright and moral rights for the publications made accessible in the public portal are retained by the authors and/or other copyright owners and it is a condition of accessing publications that users recognise and abide by the legal requirements associated with these rights.

- Users may download and print one copy of any publication from the public portal for the purpose of private study or research.

- You may not further distribute the material or use it for any profit-making activity or commercial gain

- You may freely distribute the URL identifying the publication in the public portal.

If the publication is distributed under the terms of Article 25fa of the Dutch Copyright Act, indicated by the "Taverne" license above, please follow below link for the End User Agreement:

www.tue.nl/taverne

Take down policy

If you believe that this document breaches copyright please contact us at:

openaccess@tue.nl

providing details and we will investigate your claim. 


\title{
TESLA Report 2003-04
}

\section{D Space-charge model for GPT simulations of high-brightness electron bunches}

\author{
S.B. van der Geer, O.J. Luiten \\ Eindhoven University of Technology, The Netherlands
}

\author{
M.J. de Loos \\ Pulsar Physics, The Netherlands \\ G. Pöplau*, U. van Rienen \\ Rostock University, Germany
}

\begin{abstract}
For the simulation of high-brightness electron bunches, a new 3D space-charge model is being implemented in the General Particle Tracer (GPT) code. It is based on a non-equidistant multigrid solver, allowing smooth transitions from a high to a low-aspect ratio bunch during a single run. The algorithm scales linearly in CPU time with the number of particles and the insensitivity to aspect ratio ensures that it can be used for a variety of applications. Tracking examples and field comparisons with an analytical model will be shown.
\end{abstract}

\section{Introduction}

Applications such as SASE-FELs require high quality electron bunches with an emittance of the order of 1 micron and a peak current of $1 \mathrm{kA}$ [1]. The standard method to create such bunches is to use a rf-photogun to produce relatively long bunches of several ps length, and magnetically compress them to the required current following acceleration.

A typical example of the bunch compression scheme is the first part of the DESY TTF, where a bunch of several ps is accelerated to $17 \mathrm{MeV}$ and subsequently compressed [2]. This compression stage however causes Coherent Synchrotron Radiation (CSR) degrading the transverse emittance and hence reducing bunch brightness [3].

One of the alternatives to this route are acceleration techniques based on pulsed DC technology. Attainable fields of $1 \mathrm{GV} / \mathrm{m}$ can keep a bunch sufficiently short to reach the current of $1 \mathrm{kA}$ without the need for downstream compression and thus avoiding degradation of bunch quality. An example of DC acceleration is the DC/RF scheme pioneered at Eindhoven University of Technology [4]. Here a 50 fs FWHM laser pulse is

\footnotetext{
${ }^{*}$ Supported by a research grant from DESY, Hamburg.
} 
used to create a $200 \mathrm{fs}$ electron bunch at $10 \mathrm{MeV}$ in a two-stage process. First the bunch is accelerated in a $1 \mathrm{GV} / \mathrm{m}$ field to $2 \mathrm{MeV}$. This is followed by a 2.5 cell S-band rfbooster to increase the energy to $10 \mathrm{MeV}$.

The dynamic behaviour of the space-charge fields for these approaches is very different. This can easily be seen by comparing the aspect ratio $A$ in the rest-frame of the bunch, defined as $A=R /(\gamma L)$ with $R$ the bunch radius, $L$ the length and $\gamma$ the Lorentz factor. As shown in Table A, the compression scheme has an aspect ratio of near unity, for a $1 \mathrm{~mm}$ radius, at $1 \mathrm{MeV}$. At that energy, the 'TU/e' bunch still has an aspect ratio far larger than one, i.e. a 'pancake' geometry.

Table A: Aspect ratio in the rest frame for various energies and bunchlengths measured in the lab frame.

\begin{tabular}{llll}
\hline & $0.1 \mathrm{MeV}$ & $1 \mathrm{MeV}$ & $10 \mathrm{MeV}$ \\
\hline $100 \mathrm{fs}$ & 50.85 & 11.98 & 1.62 \\
$1 \mathrm{ps}$ & 5.09 & 1.20 & 0.16 \\
$10 \mathrm{ps}$ & 0.51 & 0.12 & 0.02 \\
\hline
\end{tabular}

The remainder of this paper focuses on a new 3D space-charge model in the GPT code $[5,6]$. Section 2 shows space-charge fields for bunches with varying aspect ratios. Section 3 explains the algorithm of the new space-charge model, where the accuracy and scalability is shown in section 4 . The first tracking tests are presented in section 5.

\section{Space-charge fields}

Different aspect ratios pose different challenges to the space-charge routines in simulation codes. To demonstrate this, Figure 1 shows the transverse and longitudinal electric fields for various low aspect ratio (cigar) bunches. This is the typical regime for most electron accelerators. For very long bunches with $A<1 / 100$, the longitudinal fields become non-linear at the head and the tail of the bunch. In many simulations however, these effects can safely and conveniently be ignored because they only affect a relatively small part of the bunch and do not affect the overall results significantly.
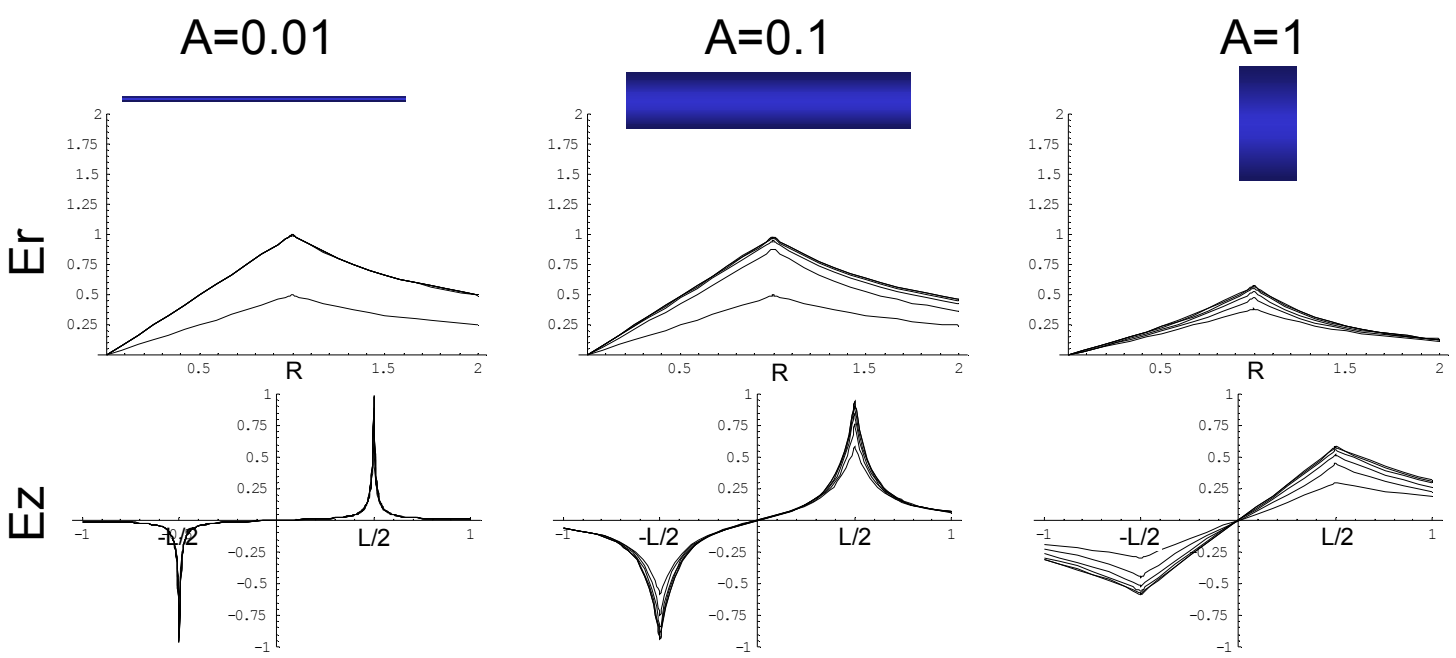

Figure 1: Transverse (top) and longitudinal (bottom) electrostatic fields in cylindrically symmetric low aspect ratio ('cigar') bunches. The different 
lines in each plot represent different positions in the bunch. All plots are created with uniform charge density.

High aspect ratio ('pancake') bunches have very different fields as shown in Figure 2. There the longitudinal fields are always relatively linear. However, when $A>100$, the transverse fields become non-linear near the edge of the bunch. This non-linearity is hard to tackle analytically and numerically because it is very ill described by a truncated Taylor expansion. In many simulations this difficult effect is ignored because bunches with an aspect ratio of 10 or more are quite uncommon in accelerators, see Table A.

For the simulation of high brightness electron bunches, where every anomaly is important, the non-linear fields in both the high and the low aspect ratio bunches need to be modelled with great precision. Especially the dynamic behaviour of the short bunches is challenging because the aspect ratio varies significantly during the first stages of acceleration.
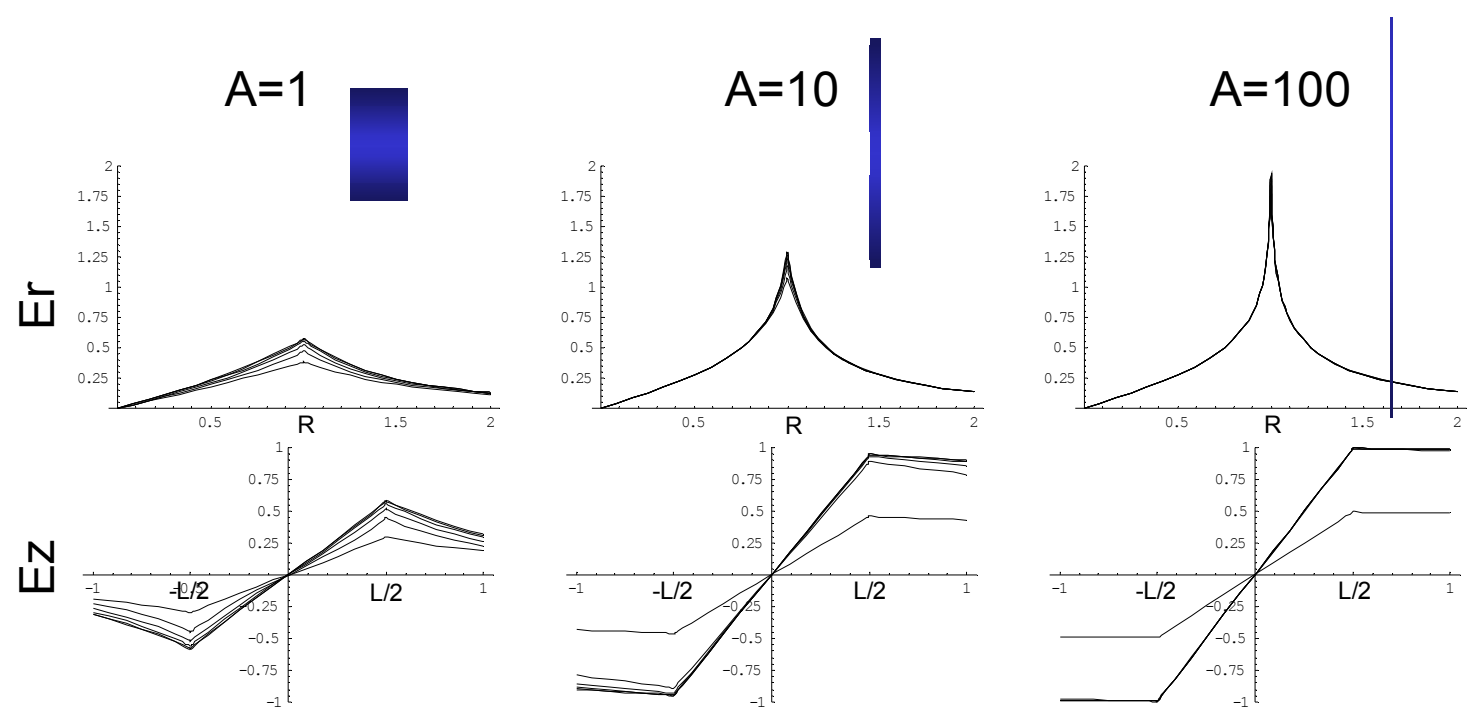

Figure 2: Transverse (top) and longitudinal (bottom) electrostatic fields in uniformly charged cylindrically symmetric high aspect ratio ('pancake') bunches. The different lines in each plot represent different positions in the bunch. All plots are created with uniformly distributed constant total charge.

\section{The new space-charge model}

\subsection{Requirements}

To be able to track high-brightness electron bunches through accelerators like the TU/e DC-RF scheme, or the DESY-TTF, the new space-charge model should be applicable to a wide parameter range in aspect ratio. At least aspect rations varying between 0.01 and 100 are required.

Furthermore, the model should be very accurate, since the design of demanding applications such as SASE-FELs relies on very accurate simulation tools. It is difficult to exactly state how accurate the model should be, but in any case the final simulation results for emittance, energy spread and bunch length must be accurate to a few percent or less. 
Because not all external fields in an accelerator are cylindrically symmetric, the spacecharge model has to be 3D. Furthermore, cathode impurities and laser anomalies often start a non-cylindrically symmetric beam on a photocathode. To simulate this process, again a $3 \mathrm{D}$ space-charge model is required.

Finally, the new space-charge routine should be fast and robust to make it useful as a design tool. The aim is to make it fast enough to allow scanning and optimisation of various parameters on a standard PC.

\subsection{Algorithm}

The chosen algorithm for the new space-charge model in GPT is a mesh-based Poisson solver in the rest-frame of the bunch. First the bunch is transformed to the frame with no average momentum. In this rest-frame, only electrostatic forces are present as long as the velocities in this frame are non-relativistic. The bunch-length in the rest-frame is longer by the Lorentz factor $\gamma$.

Subsequently, a Cartesian mesh is generated in a box around the bunch. The distribution of the meshlines is non-equidistant and adapted to the projected charge density. This is essential for the accuracy and speed of the routine. More information about the meshing is presented in section 3.3. The non-equidistant mesh is used to store an approximation of the charge density on the corners of the mesh. This information is obtained using a linear distribution of the charge of every particle over the eight corners of its enclosing meshbox.

The charge density on the mesh is fed into a Poisson solver to obtain the potential. A state-of-the-art multigrid Poisson solver [7,8] has been constructed for the nonequidistant meshes described in subsection 3.3. It scales linearly in CPU time with the number of meshnodes. Selectable Dirichlet or open boundary conditions allow the simulation of bunches within pipes with rectangular cross section, bunches near a cathode and a bunch in open space.

The resulting potential is interpolated and differentiated using a $2^{\text {nd }}$ order interpolation scheme to obtain the electric field in the rest-frame of the bunch at all particle coordinates. This electric field is transformed back to the laboratory frame to obtain the electric and magnetic fields. The GPT kernel combines these fields with the external fields in the tracking engine.

The chosen algorithm implies that the velocity in the rest-frame of the bunch is nonrelativistic. This assumption is not always true for long bunches in the first stages of acceleration. There, the situation is possible that new electrons are emitted with an energy in the eV level while the front of the bunch has already been accelerated to relativistic velocities. This is a known limitation of the routine reducing the applicability.

\subsection{Adaptive meshing}

To reduce the number of meshlines needed, an adaptive meshing technique is used. The purpose of this scheme is to both reduce 'wasted' CPU time in empty volume and reduce numerical errors by choosing more meshlines in regions with higher charge density. 
To control the 'adaptiveness' of the meshing, a control parameter $f n$ is introduced. This parameter defines the maximum difference in size between neighbouring meshnodes as shown in Figure 3. When $f n=0$, the mesh-spacing is equidistant, resulting in the most stable setting for the Poisson solver. Increasing $f n$ typically increases the overall efficiency because less nodes are required to obtain the same accuracy. A $f n$-value of $100 \%$, allowing neighbouring mesh spacing to double in size, seems to be a good compromise for a relatively large parameter range. Further increasing $f n$ typically leads to a regime where the Gauss-Seidel scheme does not always converge [9]. Obviously, different applications with different accuracy requirements and different charge density distributions have different optimal settings for $f n$.

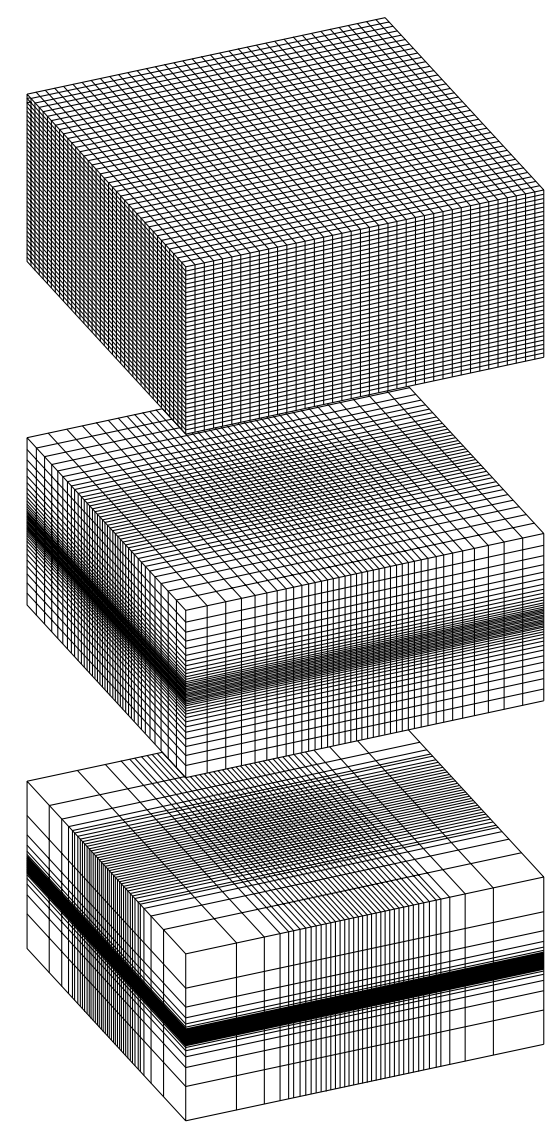

Figure 3: Different adaptive meshes for a uniformly charged hard-edge cylindrical bunch. The $f n=0$ (top) setting results in an equidistant mesh. When $f n=20 \%$ (middle) more meshlines are chosen at the position of the bunch with smooth transitions into empty space resulting in very stable solver performance. The $f n=100 \%$ case (bottom) results in very aggressive adaptive meshing, choosing almost all meshlines at the position of the bunch.

\section{Analytical test-cases of a hard-edged cylinder}

To test the accuracy of the new space-charge model, the field calculations have been compared to various analytical test cases [10]. In this section we describe the tests against analytical equations for a uniformly charged hard-edged cylindrically symmetric bunch as function of aspect ratio, number of particles and number of meshnodes. 
As shown in Figure 4, the dimensionless field error $E$ is below $10 \%$ for a range in aspect ratio varying between 0.01 and 100 for 100,000 meshnodes, where $E$ is defined as:

$$
E=\frac{\left|\mathbf{E}_{\text {analytical }}-\mathbf{E}_{\text {simulation }}\right|}{\left|\mathbf{E}_{\text {analytical }}\right|}
$$

The aspect ratios 0.01 to 10 produce much better results with $E$-values in the range of a few percent starting from 20,000 meshnodes. Although an error of a few percent may sound quite high, it should be noted that these tests represent worst-case scenarios. In actual tracking simulations, as presented in section 5, all hard-edges become smooth in a few timesteps. This produces much higher average accuracy of the space-charge routine, allowing aspect ratios over 100 and increasing the practical applicability of the algorithm beyond the range plotted in Figure 4.

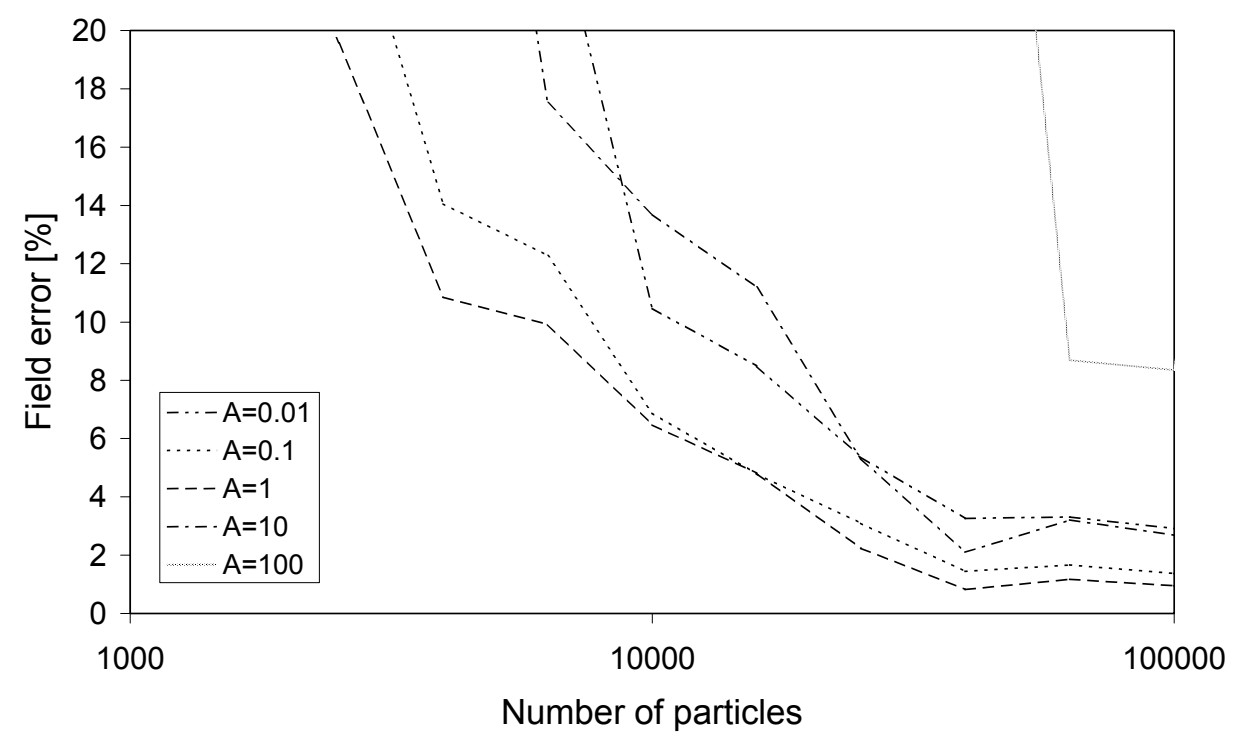

Figure 4: Field-accuracy as function of the number of particles. The number of meshnodes is chosen equal to the number of particles with $f n=100 \%$.

The Rostock multi-grid routine should ideally scale as $O(N)$ as function of number of meshnodes in terms of CPU time. As comparison, FFT based Poisson solvers scale as $O(N \log (N))$ and particle-particle interaction is an $O\left(N^{2}\right)$ process. The actual performance of the Poisson solver is very close to this ideal case, independent on aspect ratio, as shown in Figure 5. The typical scaling is as the number of meshnodes to the power 1.1. 


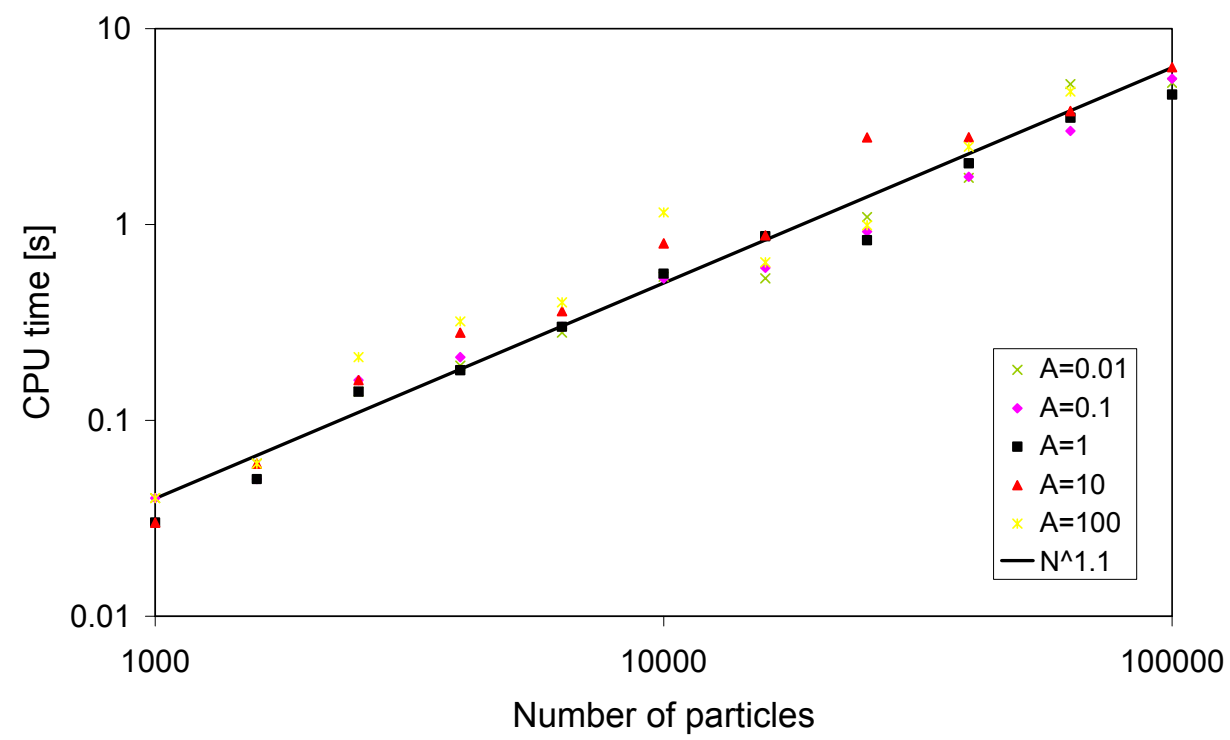

Figure 5: CPU time as function of number of particles for various aspect ratios. The number of meshnodes is chosen equal to the number of particles with $f n=100 \%$. The solid line is an exponential fit through all points and scales with the number of meshnodes to the power 1.1.

\section{Tracking tests}

\subsection{Exploding bunch}

The first tracking test of the new space-charge routine was a simulation of an exploding hard-edged cylindrically symmetric bunch during $100 \mathrm{ps}$, as shown in Figure 6. The initial bunch charge of $1 \mathrm{nC}$ is uniformly distributed over a 'pillbox' with an initial radius $R=1 \mathrm{~mm}$ and a bunchlength $L=0.1 \mathrm{~mm}$. The Lorentz factor $\gamma$ is 5 .
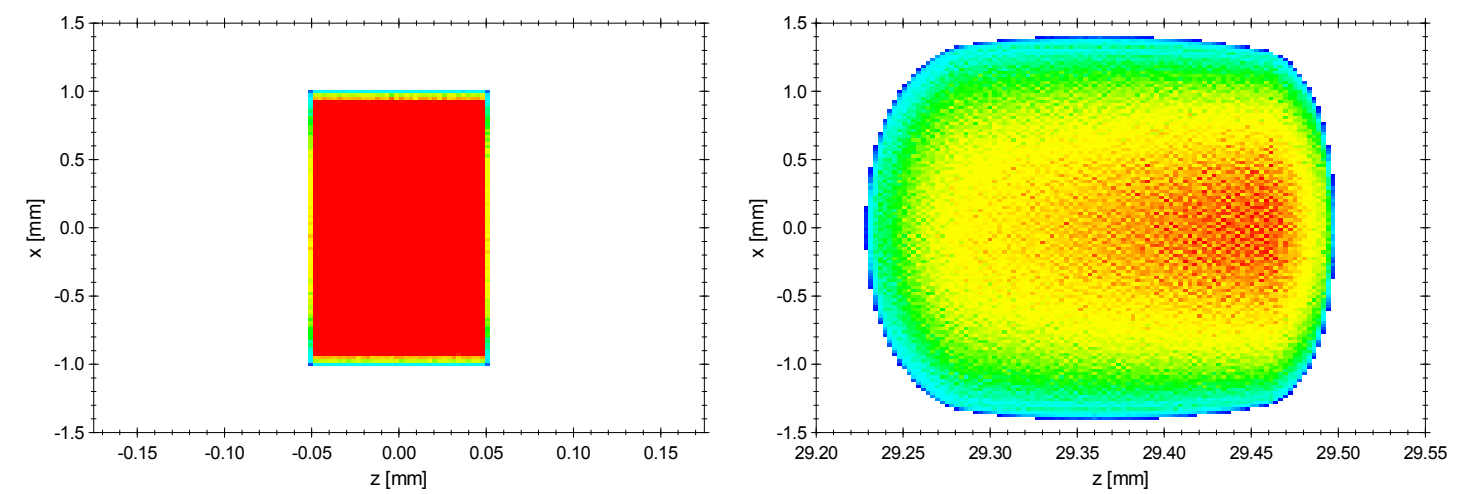

Figure 6: One million particles are tracked during $100 \mathrm{ps}$ on a mesh with 64x64x64 meshnodes.

Figure 7 shows a comparison of bunch-length and emittance of the new 3D space-charge routine with the well-tested 2D space-charge model of GPT. When the number of particles is increased, there is smooth convergence in both routines to the same values. For this specific scenario, about 10,000 particles are sufficient for convergence of the 3D model. 

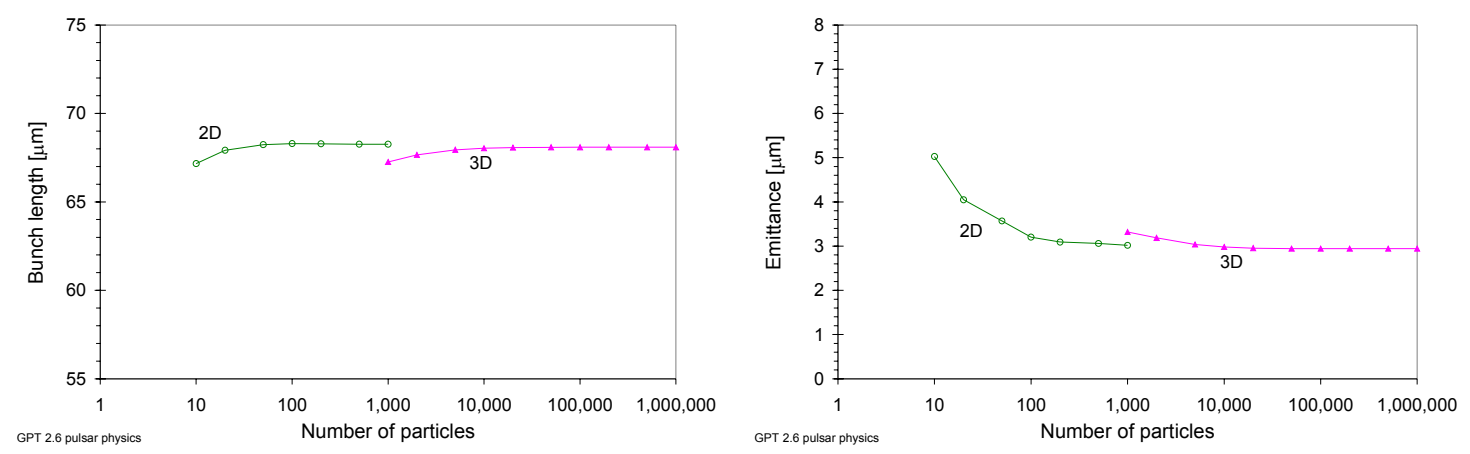

Figure 7: Final bunchlength and emittance values for an exploding $R=1 \mathrm{~mm}, 1 \mathrm{nC}$ bunch after $100 \mathrm{ps}$. Both $2 \mathrm{D}$ and 3D results are shown as function of number of particles.

\section{2. $1 \mathrm{GV} / \mathrm{m}$ diode}

To test the new space-charge routine in GPT for a scientifically relevant case, it has been used to simulate the $1 \mathrm{GV} / \mathrm{m}$ diode as is currently under construction at the Eindhoven University of Technology [11]. The set-up consisting of an anode with a circular opening and a flat cathode is shown schematically in Figure 8. During $1 \mathrm{~ns}$, a $2 \mathrm{MV}$ pulse is applied between the cathode and the anode, resulting in a DC acceleration field of $1 \mathrm{GV} / \mathrm{m}$. This field is used to accelerate an $R=0.5 \mathrm{~mm}, 100 \mathrm{pC}$ electron bunch photoextracted from the cathode surface by a 50 fs FWHM laser. Although the peak-current is over one $\mathrm{kA}$ at initiation, the bunch-quality is maintained due to the high acceleration field.

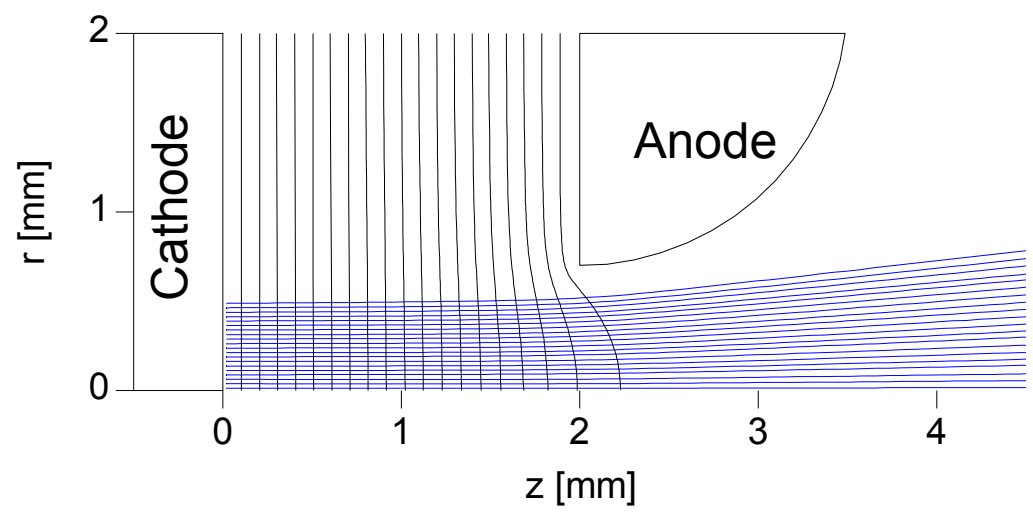

Figure 8: Schematic set-up of the $1 \mathrm{GV} / \mathrm{m}$ diode. Shown are equipotential lines and sample trajectories.

The simulation results shown in Figure 9 are a snapshot in time at $z=4.5 \mathrm{~mm}$. The large number of particles, 100,000 , produces for more detailed results compared to the previously obtained $2 \mathrm{D}$ results [11]. 

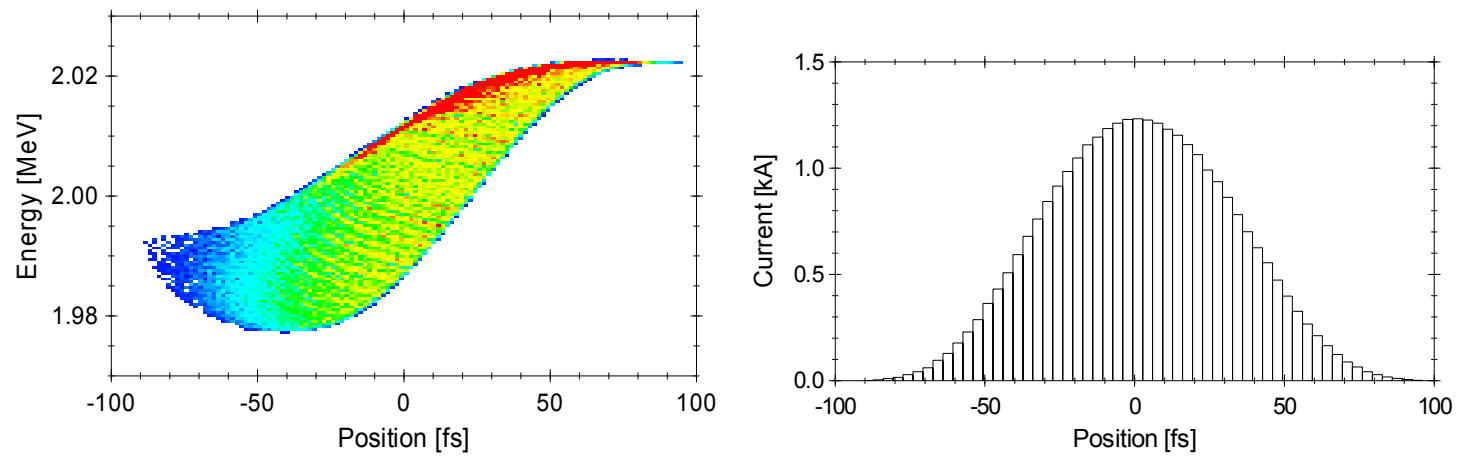

Figure 9: Raw simulation results of the $1 \mathrm{GV} / \mathrm{m}$ diode with 100,000 particles on a $60 \times 60 \times 60$ mesh with $f n=100 \%$. Shown are (left) the energy/position correlation and (right) the current.

Because the set-up is fully cylindrically symmetric, it can again be compared with the $2 \mathrm{D}$ space-charge model of GPT. Because the 2D model does not make use of a transformation to a rest-frame, the accuracy is not affected by relative energy spread. As a result, this comparison verifies that for this specific case the electrostatic assumption in the rest-frame is valid.
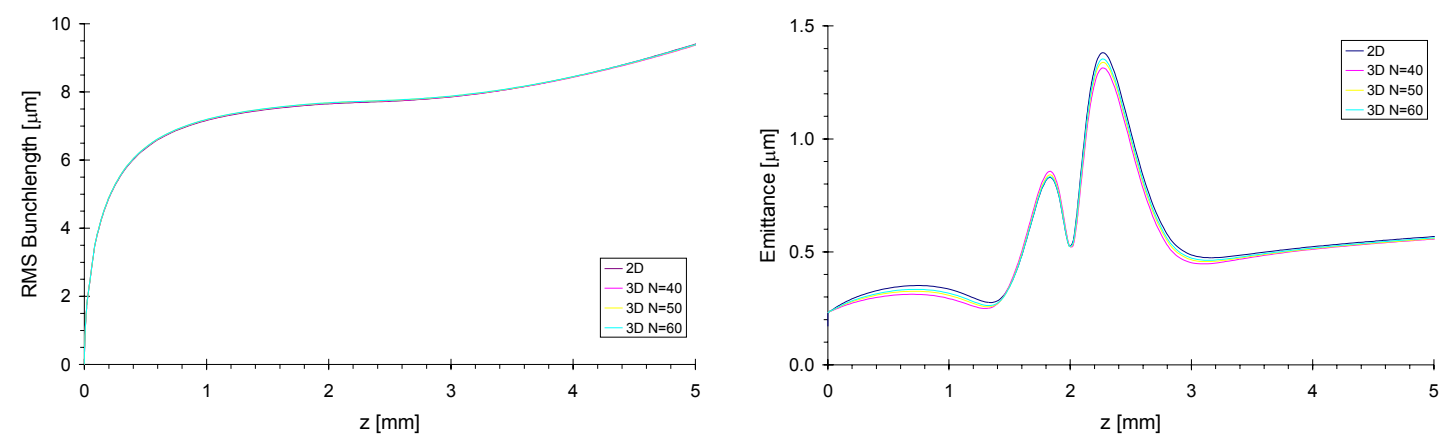

Figure 10: Comparison between the GPT 2D model and the new 3D mesh-based route applied to the TU/e $1 \mathrm{GV} / \mathrm{m}$ diode set-up. The different lines indicate different number of meshlines in the 3D model.

\section{Conclusions}

A new 3D mesh-based space-charge model has been implemented in the General Particle Tracer (GPT) code. The routine is based on a multi-grid Poisson solver to obtain the electrostatic potential in the rest frame of a charged particle bunch on a non-equidistant adaptive mesh. Lorentz transformation and interpolations are used to obtain the electromagnetic fields in the laboratory frame. The multi-grid Poisson solver scales linearly in terms of CPU time as function of the number of meshnodes over a wide range of bunch parameters.

When compared to analytical expressions for a uniformly charged cylindrically symmetric bunch, the average field error is below $10 \%$ over a range of 6 orders of magnitude in aspect ratio. Tracking tests for an exploding bunch and a $1 \mathrm{GV} / \mathrm{m}$ diode show perfect agreement with $2 \mathrm{D}$ simulation results. 
The accuracy, speed and applicability over a wide parameter range make the new routine ideally suited for the simulation of 3D space-charge effects in high-brightness electron bunches.

\section{References}

[1] M.J. van der Wiel, Conf. Proc. 2nd ICFA Future Accelerator Workshop on 'The Physics of High-Brightness Beams', Los Angeles (1999).

[2] S. Schreiber, Conf. Proc. ICFA Adv. Accel. Workshop on 'The Physics and Applications of High Brightness Electron Beams', Sardinia, Italy (2002).

[3] B. Carlsten and T. Raubenheimer, Phys. Rev. E 51, 1453 (1995).

[4] M.J. de Loos, S.B. van der Geer, F.B. Kiewiet, O.J. Luiten, M.J. van der Wiel, Proc. 2002 European Particle Accel. Conf., Paris, France, p. 1831.

[5] S.B. van der Geer, M.J. de Loos, Proc. 1998 European Particle Accel. Conf., Stockholm, Sweden, p. 1245.

[6] GPT User Manual, Pulsar Physics, De Bongerd 23, Soest, The Netherlands, www.pulsar.nl.

[7] G. Pöplau, U. van Rienen, Proc. $3^{\text {rd }}$ Conf. on Scientific Computing in Electrical Engineering 2000, edited by U. van Rienen, D. Hecht and M. Günther, LNSCE (Springer, Berlin, 2001), p. 169.

[8] G. Pöplau, U. van Rienen, M.J. de Loos, S.B. van der Geer, Conf. Proc. ICAP, Lansing, Michigan, USA (2002).

also: TESLA Report 2003-03, DESY, Hamburg, 2003.

[9] W. Hackbusch, Multi-Grid Methods and Applications, Springer, Berlin, 1985.

[10] O.J. Luiten, to be published.

[11] S.B. van der Geer, M.J. de Loos, J.I.M. Botman, O.J. Luiten, M.J. van der Wiel, Phys. Rev. E 65, 046501 (2002). 